\title{
ERÖFFNUNG DER SONDERSAMMLUNGEN KURT W. ROTH- SCHILD UND JOSEF STEINDL AN DER UNIVERSITÄTSBIBLIO- THEK DER WIRTSCHAFTSUNIVERSITÄT WIEN (WIEN, 21. OKTOBER 2014)
}

\section{von Katharina Müllebner}

Anlässlich des 100. Geburtstages des am 15. November 2010 verstorbenen Ökonomen Kurt W. Rothschild wurden am 21. Oktober 2014 die beiden Sondersammlungen Kurt W. Rothschild und Josef Steindl im Rahmen eines feierlichen Festaktes eröffnet. Im Kern bestehen die Sammlungen aus den Nachlassbibliotheken der beiden Ökonomen. Die Sammlung Rothschild wurde von Wilfried Altzinger, Institut für Makroökonomie der WU, der Universitätsbibliothek geschenkt; jene von Steindl wurde von Alois Guger, WIFO, gespendet.

Kurt W. Rothschild und Josef Steindl zählen zu den bedeutendsten österreichischen Ökonomen der 2. Republik. Sie haben nach ihrer Rückkehr aus dem britischen Exil maßgeblich zur Weiterentwicklung der ökonomische Forschung und der ökonomischen Lehre in Österreich beigetragen. Rothschild befasste sich primär mit den Fragen der Einkommensverteilung, der Lohn- und der Preistheorie, sowie mit den Fragen des Arbeitsmarktes. Steindl forschte dagegen viel zu den Themen der langfristigen Wirtschaftsentwicklung, technischen Innovationen, Bildungs- und Technologiepolitik. Beide haben als keynesianisch geprägte Ökonomen die Beschäftigung und die Kaufkraft der Bevölkerung als zentrale Elemente einer wirksamen, nachfrageorientierten Wirtschaftspolitik betrachtet ${ }^{1}$.

Die mit 200 Teilnehmer/inne/n gut besuchte Festveranstaltung wurde durch Rektor Christoph Badelt und Ingrid Kubin, Vorständin des Departments für Volkswirtschaft, eröffnet. Wilfried Altzinger führte durch den Abend und hielt einen Vortrag zu Leben und Werk von Kurt W. Rothschild, anschließend referierte Alois Guger zum Werk von Josef Steindl. Michael Katzmayr von der Universitätsbibliothek stellte die Sammlungen vor. Im Anschluss an den Vortragsteil wurde ein Dokumentarfilm² über das Leben von Kurt W. Rothschild gezeigt und Interessent/inn/en konnten die beiden Sammlungen im Rahmen von Führungen besichtigen. Beim anschließend eröffneten Buffet ergaben sich Gelegenheiten zu Diskussionen und zum 
Meinungsaustausch und bei einem Bücherstand konnten lieferbare Werke von Kurt W. Rothschild erworben werden.

\section{Die Sammlung}

Obwohl beide Nachlassbibliotheken in einem Raum untergebracht und auch nach derselben Systematik geordnet sind, handelt es sich doch um inhaltlich zwei getrennte Sondersammlungen. Diese spiegeln die vielen Gemeinsamkeiten, aber auch die Unterschiede in den Forschungsschwerpunkten der beiden Ökonomen wider, die bis heute nichts an Aktualität eingebüßt haben.

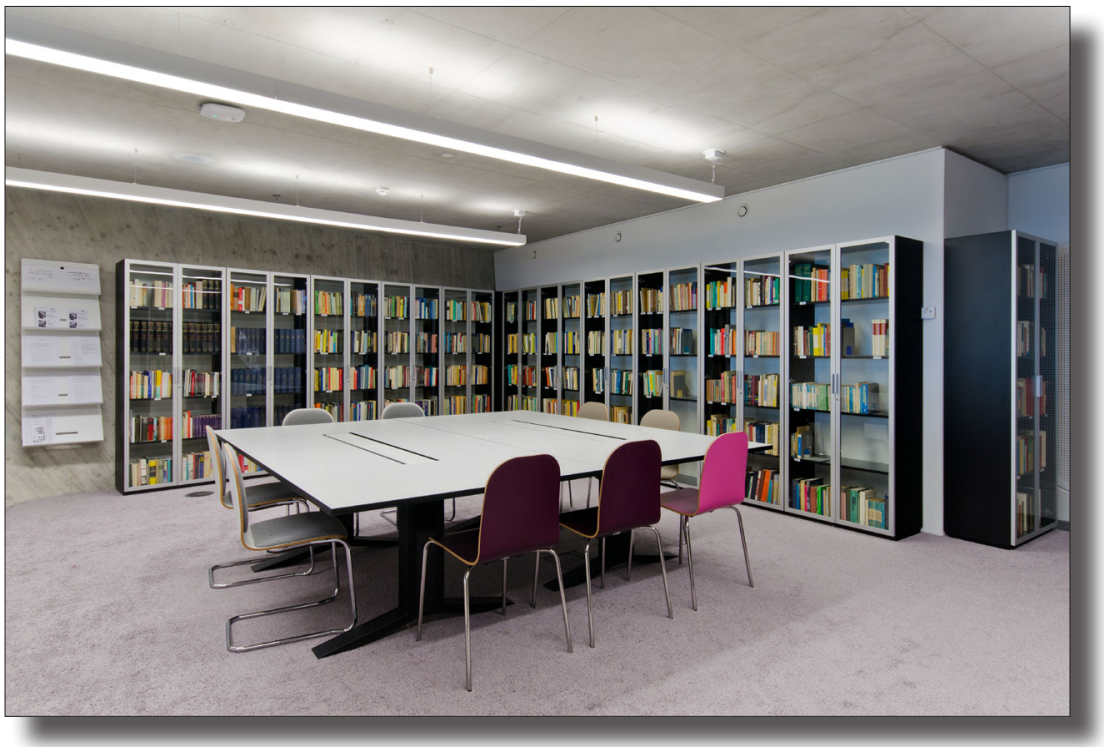

Abb. 1: Der Raum bietet auch Platz für Seminare und Gruppenarbeiten

Die rund 1.260 Werke der Sammlung Rothschild und rund 600 Werke der Sammlung Steindl sind in versperrbaren Glasvitrinen in der Universitätsbibliothek im Library and Learning Center untergebracht. Beide Sammlungen umfassen neben ökonomischer Fachliteratur auch Werke anderer Fachbereiche. Die Sammlung Rothschild beherbergt z.B. auch Bücher aus den Bereichen Soziologie, Politikwissenschaft, Geschichte und Philosophie; in der Sammlung Steindl sind die Fachbereiche Mathematik, Statistik und Naturwissenschaften z.T. stark vertreten. Die fachliche Zusammensetzung der Sammlungen sowie die Anmerkungen in vielen der Bücher lassen 
interessante Rückschlüsse auf die inhaltlichen Interessens- und Arbeitsschwerpunkte sowie methodischen Zugänge der beiden Ökonomen zu.

Die Werke sind nach einer früheren Version der Dewey Dezimalklassifikation (DDC) aufgestellt, die Kurt W. Rothschild für seine Sammlung ursprünglich verwendet hatte. Die Systematik wurde zwecks Vergleichbarkeit und einfacher Nutzbarkeit auch für die Sammlung Steindl übernommen, die nach keiner Systematik geordnet war. Besonders interessant sind die in vielen Büchern beigelegten oder darin angebrachten handschriftlichen Notizen und Materialien, etwa handschriftliche Widmungen, Rezensionsentwürfe, Korrekturen etc.

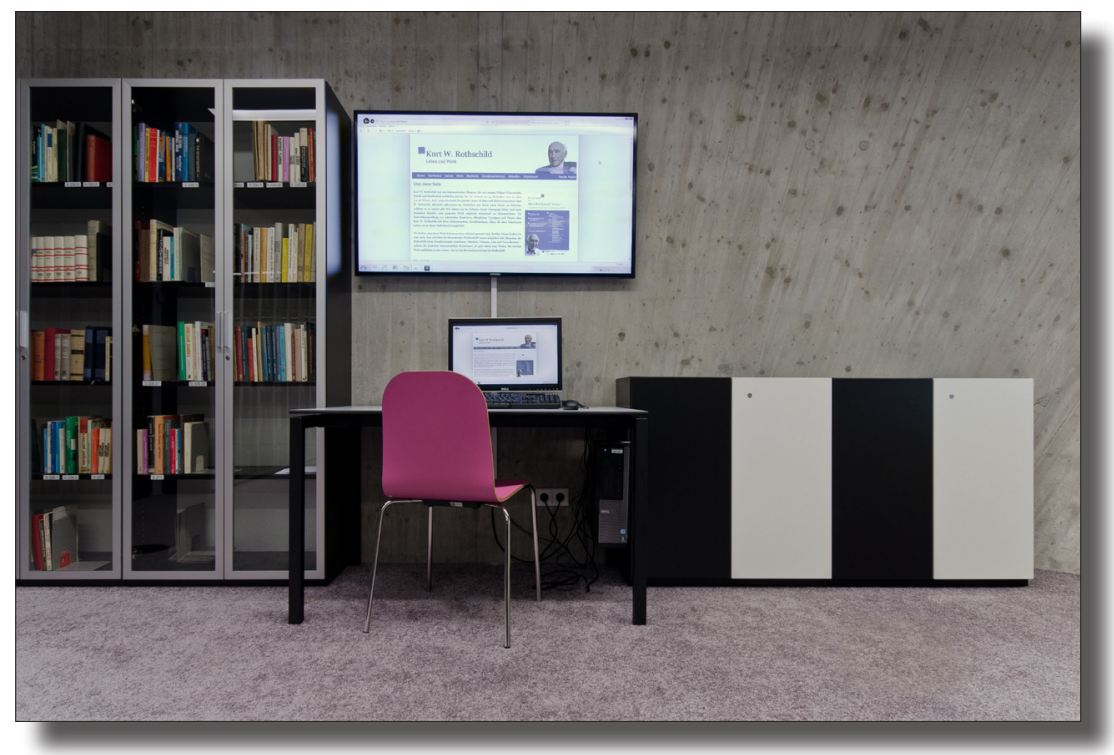

Abb. 2: Ein Computer-Arbeitsplatz samt einem Großbildschirm für Präsentationen steht zur Verfügung

\section{Nutzung}

Mit diesen beiden Sondersammlungen werden die Nachlassbibliotheken von Kurt W. Rothschild und Josef Steindl, die unzählige Ökonominnen und Ökonomen nachhaltig prägten und prägen, endlich einer breiten Fachöffentlichkeit zugänglich gemacht. Die Sammlungen sind nach vorheriger Anmeldung vor Ort benutzbar. Für weitere Informationen zu den Sammlungen und ihrer Benutzbarkeit siehe http://www.wu.ac.at/library/ service/collectionoverview/sondersammlungen-rothschild-steindl/. 
Mag. ${ }^{a}$ Katharina Müllebner Universitätsbibliothek der WU

Telefon: +43 1 31336-5724

E-Mail: katharina.muellebner@wu.ac.at

1 Ewald Walterskirchen (2014): „Zwei große österreichische Ökonomen: Kurt Rothschild und Josef Steindl."In: Die Krise verstehen. Beilage zur Wochenzeitung Falter, Nr. 34a/2014, S. 19. http://issuu.com/falter.at/ docs/o konomie/19? $=3085203 / 8986605$

2 Gerhard Teufel (2009): Wir sind Wirtschaft: Kurt W. Rothschild. Dokumentarfilm, 45 min., http://vimeo.com/21691355

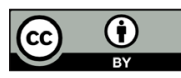

Dieses Werk ist lizenziert unter einer Creative-Commons-Lizenz Namensnennung 3.0 Österreich. 